\title{
SGLT2 inhibitors and the risk of urinary tract infections in patients with heart failure: A pooled analysis examining safety endpoints
}

\author{
Josip A Borovac ${ }^{1,2}$, Tina Ticinovic Kurir ${ }^{1,3}$, Ivona Mustapic ${ }^{2}$, Marko Kumric ${ }^{1}$, Josko Bozic ${ }^{1}$, Duska Glavas², \\ Domenico D'Amario ${ }^{4}$
}

'Department of Pathophysiology, School of Medicine, University of Split, Split, Croatia

${ }^{2}$ Clinic for Heart and Vascular Diseases, University Hospital of Split, Split, Croatia

${ }^{3}$ Department of Endocrinology and Diabetology, University Hospital of Split, Split, Croatia

${ }^{4}$ Department of Cardiovascular and Thoracic Sciences, IRCCS Fondazione Policlinico A Gemelli, Universita Cattolica Sacro Cuore, Rome, Italy

\section{Correspondence to:}

Josip A Borovac, MD, PhD

Department of Pathophysiology,

School of Medicine, University

of Split,

Soltanska 2, 21000 Split, Croatia phone: +385921721314, e-mail: jborovac@mefst.hr

Copyright by the Author(s), 2022 DOI: 10.33963/KP.a2021.0172

Received:

September 26, 2021

Accepted:

December 6, 2021

Early publication date: December 6, 2021

\section{INTRODUCTION}

Sodium-glucose cotransporter-2 inhibitors (SGLT2is) were originally envisioned as attractive hypoglycemic agents due to their promotion of glycosuria by inhibiting SGLT2 transporters in the proximal convoluted tubules of kidneys where approximately $90 \%$ of filtered glucose gets reabsorbed [1]. Due to their potent cardioprotective effects observed in trials focused on type 2 diabetes mellitus (T2DM) [2], it was hypothesized that SGLT2is might improve outcomes in heart failure (HF) patients. Indeed, it was demonstrated in landmark randomized controlled trials (RCTs) that, among patients with HF with reduced ejection fraction (HFrEF), the use of SGLT2is, compared to placebo, was associated with significant reductions in cardiovascular death and HF-related hospitalizations, both endpoints representing persistently unmet needs in HF [3]. Notably, in the DAPA-HF trial [4], patients with HF who received dapagliflozin had a $26 \%$ relative risk reduction in the composite of worsening $\mathrm{HF}$ or cardiovascular death, and results were concordant in the EMPEROR-Reduced trial [5] that evaluated the use of empagliflozin. Interestingly, robust reductions of mortality and morbidity among $\mathrm{HFrEF}$ patients were similar regardless of T2DM status at baseline. Similar trends were observed with dapagliflozin in the DECLARE-TIMI 58 sub-study [6] that was focused on a cohort of patients with T2DM and concomitant HF. On the other hand, the most recent SOLOIST-WHF [7] trial demonstrated a $33 \%$ relative risk reduction in the total number of deaths from cardiovascular causes and hospitalizations and HF-related urgent visits associated with the use of sotagliflozin vs. placebo in patients with decompensated HF. Finally, the most recent EMPEROR-Preserved trial was the first RCT that showed how a pharmacological intervention improved outcomes in patients with $\mathrm{HF}$ and preserved ejection fraction (HFpEF), as empagliflozin use was associated with a $21 \%$ relative risk reduction in a composite of cardiovascular death and hospitalizations [8].

However, post-market and surveillance studies indicated a possible association of SGLT2is and adverse events such as euglycemic diabetic ketoacidosis, genital and urinary tract infections (UTIs), Fournier gangrene, volume depletion, and limb amputations $[9,10]$. Due to their implicated glycosuric effects, susceptibility for UTIs was examined providing mixed results in patients with T2DM [11]. In large population analysis, the risk for severe or non-severe UTIs was similar among SGLT2i users compared to users of other second-line hypoglycemic drugs [12].

However, the association of SGLT2i use and UTI events has not been previously examined in the HF population on a large scale. For this reason, we performed an up-to-date analysis of five landmark RCTs evaluating the use of 
gliflozins vs. placebo in patients with HF. The main question we sought to investigate whether the risk of UTI events was increased with the use of SGLT2 is compared to placebo among patients with HF.

\section{METHODS}

Two investigators (JAB and JB) independently searched available literature in relevant databases such as PubMed and SCOPUS to include large RCTs (enrolling >1000 patients) examining the use of any SGLT2 inhibitor vs. placebo and that reported safety endpoints, such as UTI events, in the population of patients with HF. According to the PICOS (Population, Intervention, Comparator, Outcome, Study design) principle, a population of HF patients with a whole spectrum of ejection fractions (both HFrEF and HFpEF) was included. We included studies that examined the oral use of any SGLT2 inhibitor (dapagliflozin, empagliflozin, sotagliflozin) as an intervention while the comparator group received a placebo. The principal outcome of interest was the occurrence of UTI events (as reported and adjudicated by the respective study investigator committees). Due to the low number of UTI events registered in the DAPA-HF trial, we also counted events such as urosepsis, pyelonephritis, acute pyelonephritis, and staphylococcal UTI to the composite endpoint. Finally, we only considered studies that were designed and conducted as RCTs.

In short, five landmark RCTs in this setting were included, and all provided safety outcome data concerning the occurrence of UTIs. Two trials examined the use of $10 \mathrm{mg}$ dapagliflozin once-daily (DAPA-HF and DECLARE-TIMI 58), two examined the use of $10 \mathrm{mg}$ empagliflozin once-daily in HFrEF (EMPEROR-Reduced) and HFpEF (EMPEROR-Preserved), while one trial examined the use of sotagliflozin $200 \mathrm{mg}$ once daily with an eventual dose increase to $400 \mathrm{mg}$ once daily (SOLOIST-WHF). A total of 32823 patients from five RCTs were included.

\section{Statistical analysis}

The Q Cochran test and Higgins I ${ }^{2}$ statistic were calculated to estimate heterogeneity across included studies. We reported risk ratios (RR) with $95 \%$ confidence intervals (Cls) derived by using the Mantel-Haenszel random-effects statistical model. The analysis was carried out by using RevMan 5.3 (Cochrane Collaboration, London, UK). A sensitivity analysis was performed for leaving out a trial with the largest contribution to results (DECLARE-TIMI 58) to inspect if this would significantly impact the main result. The risk of bias (RoB) assessment for each trial was carried out by two investigators independently (JAB and JB), and eventual discrepancies were resolved by the third investigator (MK). The distribution of numerical variables was presented as mean (standard deviation [SD]) or median (interquartile range [IQR]). The Cochrane Collaboration's tool for assessing the risk of bias in randomized trials was used [13]. $P$-values $<0.05$ were considered statistically significant.

\section{RESULTS AND DISCUSSION}

Among 16414 patients that received SGLT2i, 585 UTI events were recorded, while 529 UTI events were recorded in 16409 patients that received a placebo. The weighted mean rate of UTI events across five landmark trials (adjusted for sample size) was 6.9 (4.1) \% in the SGLT2i group (from $0.8 \%$ to $9.9 \%$; range, 9.1 ) and 5.5 (3.2) \% in the placebo group (from $1.1 \%$ to $8.1 \%$; range, 7.0 ). Trials predominantly enrolled patients with HFrEF. The median duration of follow-up was 9.2 months in SOLOIST-WHF, 16 months in EMPEROR-Reduced, 18.2 (0-27.8) months in DAPA-HF, 26.2 (18.1-33.1) months in EMPEROR-Preserved, and finally, 50 months in DECLARE-TIMI 58. Two trials enrolled HF patients with left ventricular ejection fraction (LVEF) $<40 \%$ (DAPA-HF and EMPEROR-Reduced) while patients enrolled in SOLOIST-WHF had a median LVEF of 35 (28-46) \%. Furthermore, the EMPEROR-Preserved trial enrolled patients with HF and LVEF >40\% with a mean LVEF of 54.3 (8.8) \%. In two HFrEF cohorts, the average LVEF was 31.1 (6.8) \% in DAPA-HF and 27.5 (6.1) \% in the EMPEROR-Reduced trial. Patients with HFrEF in DECLARE-TIMI 58 (defined as those with LVEF $<45 \%$ ) had a median LVEF of $38(30-40) \%$ while those with documented HF without known reduced LVEF had a median LVEF of 55 (50-61) \%.

As shown in Figure 1, the use of SGLT2i was similar to placebo with regard to the risk of UTI events in patients with $\mathrm{HF}(\mathrm{RR}, 1.09 ; 95 \% \mathrm{Cl}, 0.94-1.26 ; P=0.24)$, and this observation was based on the evidence characterized by the low degree of heterogeneity $\left(I^{2}=25 \% ; P=0.25\right)$. Leave-one-out sensitivity analysis validated the main result (RR, 1.15; $95 \%$ $\mathrm{Cl}, 0.99-1.33 ; P=0.07)$. All trials were adjudicated as low risk of bias across all seven domains in the RoB tool.

This analysis has some limitations worth mentioning. In most of the trials, UTI events were not defined in sufficient detail, and they were not designated as events of special safety interest. Therefore, such events might be underreported, which might introduce bias with respect to the reported number of events. For example, the DAPA-HF trial reported a significantly lower number of UTI events compared to other trials since these events were not routinely collected in a pre-specified safety monitoring manner. However, in the revised analysis, we added events such as urosepsis, pyelonephritis, acute pyelonephritis, and staphylococcal UTI from this trial to the composite endpoint of UTI events. Finally, no protocol has been prospectively registered for this analysis.

Taken together, our results based on high-quality randomized trial data, show that the risk of UTI events is similar among HF patients assigned to SGLT2 inhibitor compared to those assigned to placebo, although this might be biased due to inadequate definitions and the lack of systematic registration of these events in most of the examined trials. These findings provide important safety reassurance for patients with HF, as well as for practicing cardiologists and other prescribers of this class of drugs. 


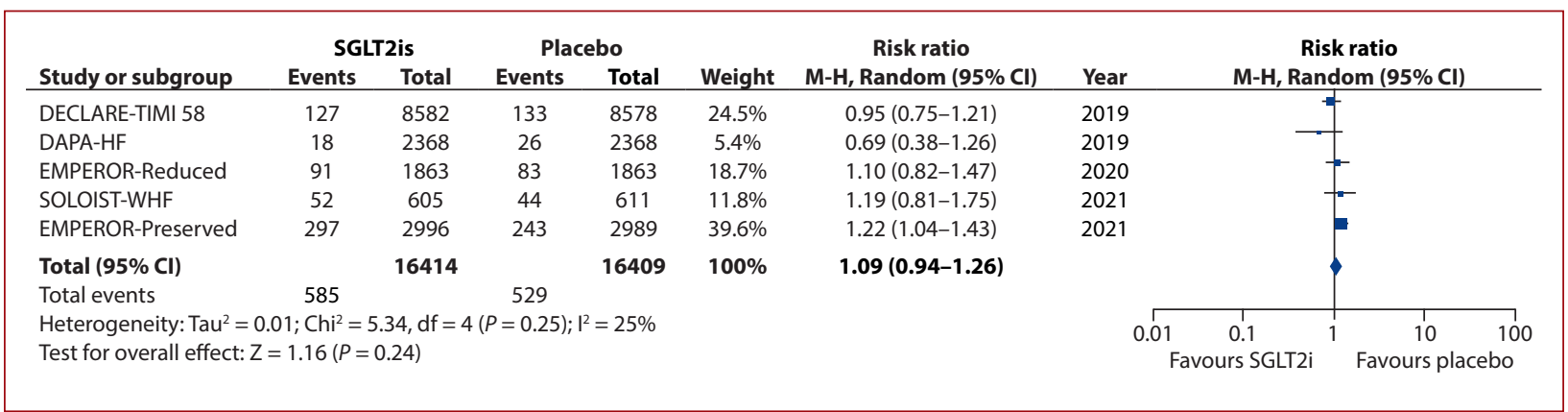

Figure 1. Results of a meta-analysis showing the relative risk of urinary tract infection events associated with SGLT2 inhibitor vs. placebo use among patients with heart failure

Abbreviations: $\mathrm{Cl}$, confidence interval; SGLT2is, sodium-glucose cotransporter-2 inhibitors; M-H, Mantel-Haenszel

\section{Article information}

Conflict of interest: None declared.

Open access: This article is available in open access under Creative Common Attribution-Non-Commercial-No Derivatives 4.0 International (CC BY-NC-ND 4.0) license, allowing to download articles and share them with others as long as they credit the authors and the publisher, but without permission to change them in any way or use them commercially. For commercial use, please contact the journal office at kardiologiapolska@ptkardio.pl.

\section{REFERENCES}

1. de Leeuw $A E$, de Boer RA. Sodium-glucose cotransporter 2 inhibition: cardioprotection by treating diabetes - A translational viewpoint explaining its potential salutary effects. Eur Heart J Cardiovasc Pharmacother. 2016; 2(4): 244-255, doi: 10.1093/ehjcvp/pvw009, indexed in Pubmed: 27533948.

2. Kluger AY, Tecson KM, Lee AY, et al. Class effects of SGLT2 inhibitors on cardiorenal outcomes. Cardiovasc Diabetol. 2019; 18(1): 99, doi: 10.1186/s12933-019-0903-4, indexed in Pubmed: 31382965.

3. Nessler J, Siniarski A, Leszek P, et al. Reviewers. Expert opinion of the Heart Failure Working Group of the Polish Cardiac Society on the use of dapagliflozin in the treatment of heart failure with reduced ejection fraction. Kardiol Pol. 2021; 79(3): 363-370, doi: 10.33963/KP.15859, indexed in Pubmed: 33687868.

4. McMurray J, Solomon S, Inzucchi S, et al. Dapagliflozin in patients with heart failure and reduced ejection fraction. N Engl J Med. 2019; 381(21): 1995-2008, doi: 10.1056/nejmoa1911303, indexed in Pubmed: 31535829.

5. Anker SD, Butler J, Filippatos G, et al. Cardiovascular and renal outcomes with empagliflozin in heart failure. N Engl J Med. 2020;383(15): 1413-1424, doi: 10.1056/NEJMoa2022190, indexed in Pubmed: 32865377.
6. Kato ET, Silverman MG, Mosenzon O, et al. Effect of dapagliflozin on heart failure and mortality in type 2 diabetes mellitus. Circulation. 2019; 139(22):2528-2536, doi: 10.1161/CIRCULATIONAHA.119.040130, indexed in Pubmed: 30882238.

7. Szarek M, Bhatt DL, Steg PhG, et al. Sotagliflozin in patients with diabetes and recent worsening heart failure. N Engl J Med. 2021; 384(2): 117-128, doi: 10.1056/NEJMoa2030183, indexed in Pubmed: 33200892.

8. Butler J, Filippatos G, Siddiqi TJ, et al. Evaluation of the effects of sodium-glucose co-transporter 2 inhibition with empagliflozin on morbidity and mortality in patients with chronic heart failure and a preserved ejection fraction: rationale for and design of the EMPEROR-Preserved Trial. Eur J Heart Fail. 2019; 21(10): 1279-1287, doi: 10.1002/ejhf.1596, indexed in Pubmed: 31523904.

9. Singh $M$, Kumar A. Risks associated with SGLT2 inhibitors: an overview. Curr Drug Saf. 2018; 13(2): 84-91, doi: 10.2174/15748863136661802261 03408, indexed in Pubmed: 29485006.

10. Bersoff-Matcha SJ, Chamberlain C, Cao C, et al. Fournier gangrene associated with sodium-glucose cotransporter-2 inhibitors: a review of spontaneous postmarketing cases. Ann Intern Med. 2019; 170(11): 764-769, doi: 10.7326/M19-0085, indexed in Pubmed: 31060053.

11. Puckrin R, Saltiel MP, Reynier P, et al. SGLT-2 inhibitors and the risk of infections: a systematic review and meta-analysis of randomized controlled trials. Acta Diabetol. 2018; 55(5): 503-514, doi: 10.1007/s00592-018-11160 , indexed in Pubmed: 29484489.

12. Dave CV, Schneeweiss S, Kim D, et al. Sodium-Glucose cotransporter-2 inhibitors and the risk for severe urinary tract infections: a population-based cohort study. Ann Intern Med. 2019; 171(4): 248-256, doi: 10.7326/M183136, indexed in Pubmed: 31357213.

13. Higgins JPT, Altman DG, Gøtzsche PC, et al. The Cochrane Collaboration's tool for assessing risk of bias in randomised trials. BMJ. 2011; 343: d5928, doi: 10.1136/bmj.d5928, indexed in Pubmed: 22008217. 